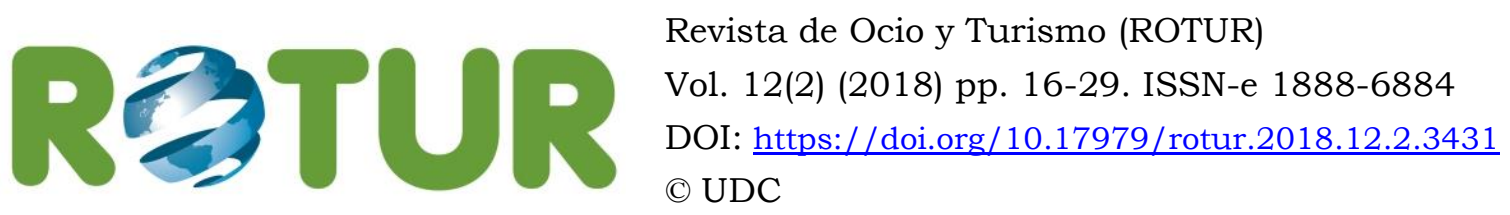

\title{
Identidad patrimonial e implicación local en el desarrollo turístico de Lorca (Región de Murcia, España)
}

\section{Patrimonial identity and local implication in the touristic development of Lorca (Murcia Region, Spain)}

Daniel Moreno Muñoz
Universidad de Murcia
daniel.moreno1@um.es

Ramón García Marín Universidad de Murcia ramongm@um.es

Cayetano Espejo Marín Universidad de Murcia cespejo@um.es

Recibido/Received: 18/04/2018 Aceptado/Accepted: 24/07/2018

\section{RESUMEN:}

Lorca sufrió en 2011 dos seísmos que ocasionaron 9 víctimas mortales y daños materiales en viviendas y en su patrimonio histórico. Desde entonces parece apostar por el desarrollo del turismo cultural como actividad para su progreso socioeconómico y la revitalización de su casco histórico. Para lograr este cometido resulta necesaria la implicación de los actores locales; una población que conozca y valore su identidad, patrimonio y cultura para poder venderla de la mejor forma posible. ¿Existe una identidad cultural y patrimonial construida y reforzada recientemente para poder dar respuesta a las exigencias de turistas y visitantes? $¿$ Convive en la ciudad una complicidad por parte de los actores locales para que este proyecto se convierta en una realidad? En esta investigación se responderá a las preguntas planteadas.

Palabras clave: Identidad Patrimonial; Implicación Local; Turismo Cultural; Desarrollo Local; Lorca.

\section{ABSTRACT:}

The city of Lorca suffered in 2011 two earthquakes that caused 9 fatalities and material damage to homes and their historical heritage. Since then it seems to bet on the development of cultural tourism as an activity for its socio-economic progress and the revitalization of its historic center. To achieve this goal, the involvement of local actors is necessary; a population that knows and values it's identity, heritage and culture in order to sell it in the best possible way. Is there a cultural and patrimonial identity recently built and reinforced in order to respond to the demands of tourists and visitors? Is there a complicity on the part of local 
actors in the city for this project to become a reality? In this investigation, the questions posed will be answered.

Keywords: Patrimonial Identity; Local Implication; Cultural Tourism; Local Development; Lorca

\section{INTRODUCCIÓN}

La identidad de las personas, a menudo, se explica en términos de nacionalidad. No obstante, este concepto puede abarcar diferentes aspectos como actitudes, valores, tradiciones, mitos o incluso el patrimonio (Bowitz e Ibenholt, 2009). La identidad, el patrimonio y el turismo están conectados por serie de vínculos sociales, económicos y políticos conformados con el paso del tiempo. Actualmente, existe un fuerte interés político en el patrimonio cultural (Burghausen y Balmer, 2014). Durante la mayor parte del siglo XX, la preservación de éste tuvo como objetivo restaurar y mantener las "piedras" tangibles de un entorno históricamente significativo (Zukin, 2012). No obstante, desde el decenio de 1980, se comienza a incluir un objetivo diferente: mantener vivo el "espíritu" intangible de los pequeños grupos sociales, cuyas costumbres y habilidades estaban quedando marginadas por la sociedad moderna (Vecco, 2010).

Cada territorio ha adquirido una idiosincrasia propia que lo hace singular. Cuentan con unos recursos patrimoniales, materiales e inmateriales, que han sido interiorizados por sus habitantes en su modo de vida, costumbres y tradiciones. La identidad de los ciudadanos está ligada al patrimonio, teniendo relación con la conservación de éste (Henderson, 2010). Esto se reproduce desde el pasado con proyección de futuro, guardando simbolismos que contienen en sí mismos un sentido de visión hacia el desarrollo y la promoción social comunitaria (Bruzón, 2017).

Conviene advertir que hay manifestaciones culturales que expresan con mayor intensidad que otras su sentido de identidad, hecho que las diferencia de otras actividades que son parte común de la vida cotidiana (Molano, 2007). Por ello, diversas expresiones religiosas, tipos de música o bailes, que tienen una gran repercusión territorial, la UNESCO las ha registrado bajo el concepto de patrimonio cultural inmaterial.

Una de las vias de desarrollo en las ciudades históricas es la puesta en valor de la identidad territorial. Es decir, muestran una serie de valores locales que han formado parte de la historia de la localidad.

El binomio patrimonio y turismo se combinan, se complementan y se necesitan (Prats, 2003). Las ciudades patrimoniales se encuentran ante la disyuntiva y necesidad de hacer frente a varios retos tendentes a favorecer el desarrollo local, destacando los siguientes i) aprovechar el crecimiento constante de la cultura en los desplazamientos turísticos; ii) alcanzar la revitalización económica en los centros históricos; y iii) conseguir la recuperación y puesta en valor de ricos bienes arquitectónicos. Troitiño y Troitiño (2016) manifiestan que el patrimonio cultural, en sus diversas expresiones, tiene potencialidades diversas de utilización turística, pero, para rentabilizarlas, hay que disponer no solo de un buen conocimiento de la realidad y la gestión turística de los destinos, sino también de la dimensión turística de los recursos patrimoniales, sean monumentos, espacios urbanos, fiestas o tradiciones. Desde el punto de vista económico, el patrimonio es esencial por su papel en el turismo y en el desarrollo local (Capel, 2014), siendo utilizado por la comunidad anfitriona como un recurso. De acuerdo con Cebrián (2001), el turismo cultural constituye una salida viable, siempre y cuando se parta del encaje de la nueva actividad en la sociedad receptora. Es decir, los actores locales deben implicarse y jugar un papel decisivo a la hora de poner en marcha la actividad.

El turismo es un modo de acceder a la cultura (Richards, 1996), incluida la local, con sus señas de identidad que se han forjado a lo largo de la historia. La identidad es resultado de un hecho objetivo (el determinante geográfico-espacial, los datos históricos y las específicas 
condiciones socioeconómicas) y una construcción de naturaleza subjetiva (la dimensión metafísica de los sentimientos y los afectos, la propia experiencia vivencial, la conciencia de pertenencia a un universo local o de otro nivel de integración sociocultural, la tradición, el capital cultural y la específica topografia mental que representan rituales, símbolos y valores) (Marcos, 2004). Por ejemplo, las actividades artesanas forman parte de la identidad colectiva y su contribución a la preservación del acervo cultural y a la riqueza etnológica.

La situación en la que hoy está la ciudad de Lorca, seis años después de las desgracias ocasionadas por los seísmos, aconsejan inculcar en el ambiente urbano que el desarrollo local no puede entenderse como un procedimiento dependiente de las decisiones políticas que en cada momento convenga difundir, sino que debe ser asumido como un proceso que debe sustentarse en experiencias locales sólidas, y para ello nada mejor que otorgar prioridad a la cultura local (García et al., 2016), entendida como el saber hacer de las personas en su vida cotidiana (Abdelmalki y Courlet, 1996). Con el reciente énfasis del desarrollo territorial se observa un período de rescate y valoración de la tradición cultural y los vínculos con determinados territorios apelando a la pretendida identidad territorial. En este sentido, en el marco de un trabajo de investigación, parece oportuno poner en evidencia la identidad local que se ha forjado a través de los distintos elementos patrimoniales existentes en la ciudad simbiosis sinérgica que pueden generar estos dos factores imprescindibles para el desarrollo de una ciudad histórica. Los principales objetivos de esta investigación han sido (1) Averiguar la identidad patrimonial de los ciudadanos lorquinos; (2) Conocer la implicación de los actores locales respecto al desarrollo del turismo cultural; y (3) Estudiar la incidencia que el turismo cultural ha tenido en el desarrollo de la ciudad.

\section{METODOLOGÍA}

Para la elaboración de la presente investigación se realizó, en primer lugar, una revisión bibliográfica exhaustiva del tema en cuestión. Seguidamente, se recopiló la información disponible acerca de la oferta turística cultural en Lorca, así como los planes de la administración local (pero también regional) en materia de dinamización turística, pues para abordar un estudio de este tipo es necesario conocer cuáles son las políticas actuales a seguir en el ámbito de análisis. Para la cuantificación de visitas recibidas por la ciudad y la Fortaleza del Sol se consultaron fuentes estadísticas locales, proporcionadas por el Consorcio Turístico Lorca Taller del Tiempo, y regionales, procedentes de la Comunidad Autónoma de la Región de Murcia a través de Murcia Turistica.

Por último, se efectuó un trabajo de campo con el fin de comprobar in situ los elementos patrimoniales existentes en Lorca que han sido convertidos en recursos turísticos. Además, se mantuvieron 130 entrevistas distendidas con los distintos actores locales (ciudadanos, comerciantes, técnicos especializados y miembros de la corporación municipal) lorquinos con el fin de constatar su percepción sobre el patrimonio que consideran parte de su identidad y en qué grado están implicados en el desarrollo del turismo cultural en la ciudad.

\section{III. ÁREA DE ESTUDIO}

Lorca se localiza en el sureste español, concretamente en la Región de Murcia. Su término municipal abarca una extensión total de $1.675,2 \mathrm{~km}^{2}$, siendo el segundo municipio en extensión de España tras Cáceres. Limita al Norte con los municipios de Caravaca de la Cruz y Cehegín, al Este con los de Mula, Aledo, Totana y Mazarrón, al Sur con Águilas y el Mediterráneo y al Oeste con los términos almerienses de Vélez Blanco, Vélez Rubio, HuércalOvera y Pulpí. Actualmente cuenta con 91.730 habitantes (INE, 2016), de los que 58.866 viven en la ciudad de Lorca, consolidándose ésta como la tercera en importancia dentro del contexto 
regional, tras Murcia y Cartagena. La comarca natural de Lorca es más amplia, alcanzando los $3.970 \mathrm{~km}^{2}$, teniendo la ciudad influencia directa sobre otras diez localidades distribuidas casi a la par entre las provincias de Murcia y Almeria, quedando este espacio poblado por 211.534 habitantes (Serrano et al., 2016).

En la ciudad se han asentado diversas civilizaciones a lo largo del tiempo debido, primordialmente, a su particular ubicación en el extremo oriental de la depresión penibética, camino obligado entre Andalucía y Levante (Gil, 1968). Forma parte de un extenso corredor que comunica los históricos reinos de Murcia y Granada, siendo una de las ciudades defensivas y fronterizas entre ambos (Martínez, 1985).

Posee un clima benigno a lo largo de todo el año, caracterizado por unas temperaturas medias relativamente elevadas $\left(18,1^{\circ} \mathrm{C}\right)$ y una baja pluviosidad $(261 \mathrm{~mm}$ en la ciudad, concentrados en apenas 25 días), lo que genera unos marcados rasgos de aridez que inciden en la vegetación (apenas la tercera parte del territorio es forestal), en el asentamiento de la población y en el desarrollo de las actividades económicas (Romera, 2016).

\section{RECURSOS PATRIMONIALES, CULTURA E IDENTIDAD TERRITORIAL EN LORCA}

Su milenaria historia ha conferido a Lorca una serie de elementos patrimoniales que revelan el pasado vivido por la ciudad. Son elementos que reflejan los hitos que la han configurado y, por normal general, hacen referencia a periodos históricos de esplendor. El más destacado es la Fortaleza del Sol o Castillo de Lorca. Se alza sobre un cerro con óptimas condiciones de seguridad y control sobre el valle del Guadalentín, circunstancias que favorecieron el establecimiento de población desde la prehistoria. Se trata de la seña de identidad de la ciudad y un símbolo para los lorquinos, pues constituyó un bastión defensivo que marcó durante siglos la frontera entre el Reino de Murcia y el nazarí de Granada. Actualmente se puede visitar todos los días del año, encontrando propuestas culturales muy diversas, con exposiciones permanentes y temporales, visitas guiadas y espacios de participación y ocio. En época estival, con el fin de atraer visitantes, se realizan época estival actividades nocturnas como conciertos o rutas teatralizadas a la fortaleza. El grado de aceptación está siendo más que evidente, así lo reflejan los datos. Entre julio y septiembre de 2017 se registraron 12.824 visitantes, 7.687 más que en el año anterior. Es decir, la evolución en la última anualidad ha sido muy fructífera.

Además de la citada Fortaleza del Sol es provechoso mencionar otros monumentos que conforman el patrimonio de la ciudad y que son visitados por los viajeros. Destacan entre otros: la Ex Colegiata San Patricio, el Teatro Guerra, la Casa Guevara, la Iglesia de Santo Domingo, el Casino, los escudos nobiliarios, la Iglesia de San Francisco o el propio Ayuntamiento.

Por otro lado, a lo largo de la historia han sido múltiples los oficios artesanales presentes en el municipio de Lorca (jarapas, forja o alfareria), especialmente en el medio rural en zonas como el denominado Campo de Lorca o la zona de las Tierras Altas. Diversas actividades han perdurado hasta la actualidad con cierta relevancia en la economía lorquina, siendo conocidas a escala regional, nacional e incluso internacional. Pese a los cambios tecnológicos han mantenido su identidad artesanal, valorizando aún más si cabe el producto elaborado. Son disciplinas transmitidas, en la mayoría de los casos, de generación en generación, una clara manifestación de conocimientos tácitos.

La Dirección General de Artesanía creó tres espacios dedicados a los oficios artesanales. Se sitúan en las ciudades de mayor importancia en el contexto regional (Murcia, Cartagena y Lorca). Se trata, por tanto, de lugares que sirven como instrumento necesario para potenciar el sector mediante la comercialización de productos, realización de exposiciones, jornadas de formación y demostraciones. El centro lorquino se localiza en pleno corazón de la ciudad, en la 
transitada calle Lope Gisbert, entre la Iglesia de San Mateo y el Palacio de Guevara. La vertebración turística de la ciudad en torno al turismo cultural, confieren una importancia sustancial a este espacio, dotando a Lorca de una singularidad competitiva frente a otras localidades próximas de la misma Región y de provincias limítrofes.

En las encuestas realizadas, los ciudadanos lorquinos han demostrado un gran conocimiento de la diversidad patrimonial existente. Llama la atención que se destaquen los elementos localizados en el centro histórico de la ciudad, obviando otros recursos existentes en el territorio municipal. Además, las manifestaciones están relacionadas con edificios religiosos, especialmente con las iglesias.

Conviene advertir sobre el patrimonio intangible. Éste no ha sido señalado por ninguno de los entrevistados, ciñéndose solamente al tangible. No obstante, es curioso, como se verá más adelante, que un recurso con una componente material e inmaterial como son los bordados, identificado como la principal seña de identidad del municipio, no sea indicado como un elemento patrimonial a pesar de existir en la ciudad cuatro museos destinados a la exposición de las piezas bordadas y una fuerte campaña publicitaria con la palabra bordado.

Si se analizan las respuestas de una manera más detallada, el Castillo es el recurso patrimonial más señalado por los ciudadanos con un 98,5 \% de las evidencias (Imagen 1). Tras él se sitúan la Iglesia de Santo Domingo (88,5 \%), la Ex - Colegiata San Patricio (80,8 \%) y la Casa Guevara $(78,5 \%)$ como otros elementos destacados dentro del patrimonio de la ciudad. Además, otros lugares como la Iglesia de Santiago, el Huerto Ruano, la Iglesia de Nuestra Señora del Carmen o el Santuario Virgen de las Huertas, también han sido citados pero con porcentajes bastante inferiores.

Imagen 1. Castillo de Lorca

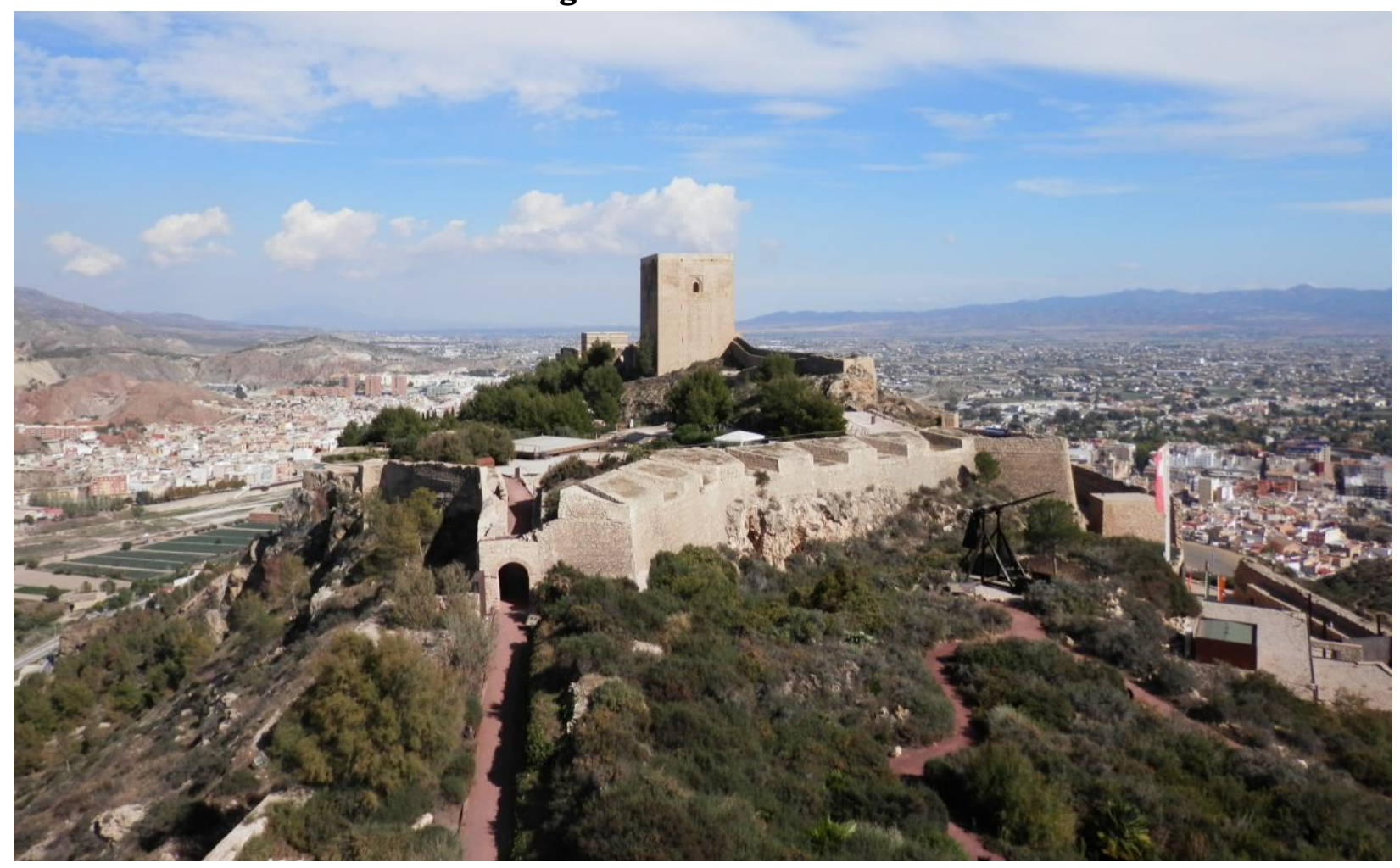

Fuente: Autores.

Para los encuestados los bordados se conforman como seña de identidad del pueblo lorquino, habiendo sido manifestado por el $94,6 \%$. Le siguen en importancia el Castillo (80,8 \%) y la Semana Santa $(76,2 \%)$, festividad que tiene una relación directa con los bordados, pues éstos 
forman parte de la misma. Con valores más distantes se sitúan las dos actividades económicas tradicionales de Lorca, agricultura y ganaderia, y la gastronomía. Por tanto, el Castillo es el único elemento patrimonial es destacado como parte de la idiosincrasia lorquina.

El $83,4 \%$ de los encuestados manifestaron que existe una propia identidad patrimonial en Lorca. Mientras que el 16,6 \% revelan lo contrario. Es decir, existe una aceptación entre los lorquinos en la relación del patrimonio con la identidad territorial. A pesar de ello, las opiniones son diversas:

"Lorca y los lorquinos tenemos una identidad patrimonial propia. El patrimonio es variado y forma parte de nuestro modo de vida. Nuestro elemento más característico son los bordados, una técnica que se realiza en la ciudad desde hace más de 100 años y perdura en la actualidad. Por otro lado, el Castillo lo llevamos muy interiorizado, pues es visible desde muchas partes de la ciudad, siendo una de sus peculiaridades".

"No creo que exista una identidad patrimonial propia, todo está ligado al patrimonio de la Región de Murcia, pues las caracteristicas son similares. No obstante, creo que la ciudad tiene un simbolo identificativo bastante evidente que es la Fortaleza del Sol, pues su silueta forma parte de las campañas publicitarias existentes desde hace décadas".

Por último, el 74,2 \% considera que la identidad patrimonial de la ciudad es uno de los factores en los que se apoya el desarrollo turístico de la ciudad, especialmente tras los terremotos del 11 de mayo de 2011. Por el contrario, el $17,1 \%$ señala que no es influyente a la hora de promocionar el turismo de la ciudad, mientras que para el 8,7 \% le resulta indiferente.

En sintesis, los lorquinos tienen diversos elementos patrimoniales como parte de su identidad. Ésta se ha ido conformando con el tiempo, es decir, se trata de una construcción social. Además, actualmente tiene un papel relevante en el desarrollo turístico de la ciudad, pues es empleada en la promoción turística de manera constante.

\section{LOS BORDADOS COMO PARTE DE LA IDENTIDAD TERRITORIAL}

Tal como han manifestado los encuestados, los bordados que realizan los distintos pasos que conforman la Semana Santa lorquina son una de sus grandes señas de identidad. Por ello, se hace preciso indagar sobre los mismos.

Una de las singularidades más atractivas de la Semana Santa lorquina son sus bordados. Éstos se conforman como un patrimonio material e inmaterial, pues el arte y la técnica del bordado se transmiten de generación en generación por las costureras de los diversos pasos que participan en la Semana Santa (Espejo y García, 2016), siendo una clara manifestación de conocimientos tácitos. El bordado en oro y sedas constituye el componente esencial de los Desfiles Bíblico-Pasionales de Lorca (Imagen 2). Su materialización es fruto de la implicación directa de muchos lorquinos que durante todo el año se esfuerzan para hacer realidad este objetivo común. El arte del bordado lorquino es, en suma, una manifestación artística que permite a los lorquinos expresar su creatividad y un símbolo reconocido de su identidad cultural (Moreno et al., 2017).

Para mantener viva la tradición y su transmisión de generación a generación, se ha creado en la colaboración de diferentes organismos públicos una escuela oficial de bordado en el que el alumnado aprende las técnicas que les enseñan bordadoras y directores artísticos. La transmisión de estos conocimientos y prácticas ha permitido el aprendizaje y desarrollo de estas técnicas, favoreciendo su popularización y el establecimiento de vínculos sociales.

Tras los terremotos, las cofradias que compone la Semana Santa deciden sus museos expositivos. Estas instalaciones albergan un rico y variado patrimonio. Los más concurridos son los del Paso Blanco y el Paso Azul, siendo visitados por miles de personas cada año. Son 
una muestra inequívoca de la pasión con la que es vivida la festividad en la ciudad y un fiel reflejo de uno de los elementos idiosincráticos del pueblo lorquino.

Actualmente existe una propuesta para incluir a los bordados de Lorca en la Lista Representativa del Patrimonio Inmaterial de la Humanidad por la UNESCO. Se materializa así un deseo cuyo origen se encuentra años atrás, cuando los pasos solicitan, en primer lugar, la máxima protección jurídica de algunas de las piezas de bordados a través de la figura de Bien de Interés Cultural. Esta llega mediante el Decreto n. ${ }^{\circ}$ 5/2014, de 14 de febrero, del Consejo de Gobierno de la Comunidad Autónoma de la Región de Murcia, por el que se declara Bien de Interés Cultural inmaterial a favor de "La Tradición del Bordado de Lorca".

Imagen 2. Bordado lorquino en detalle

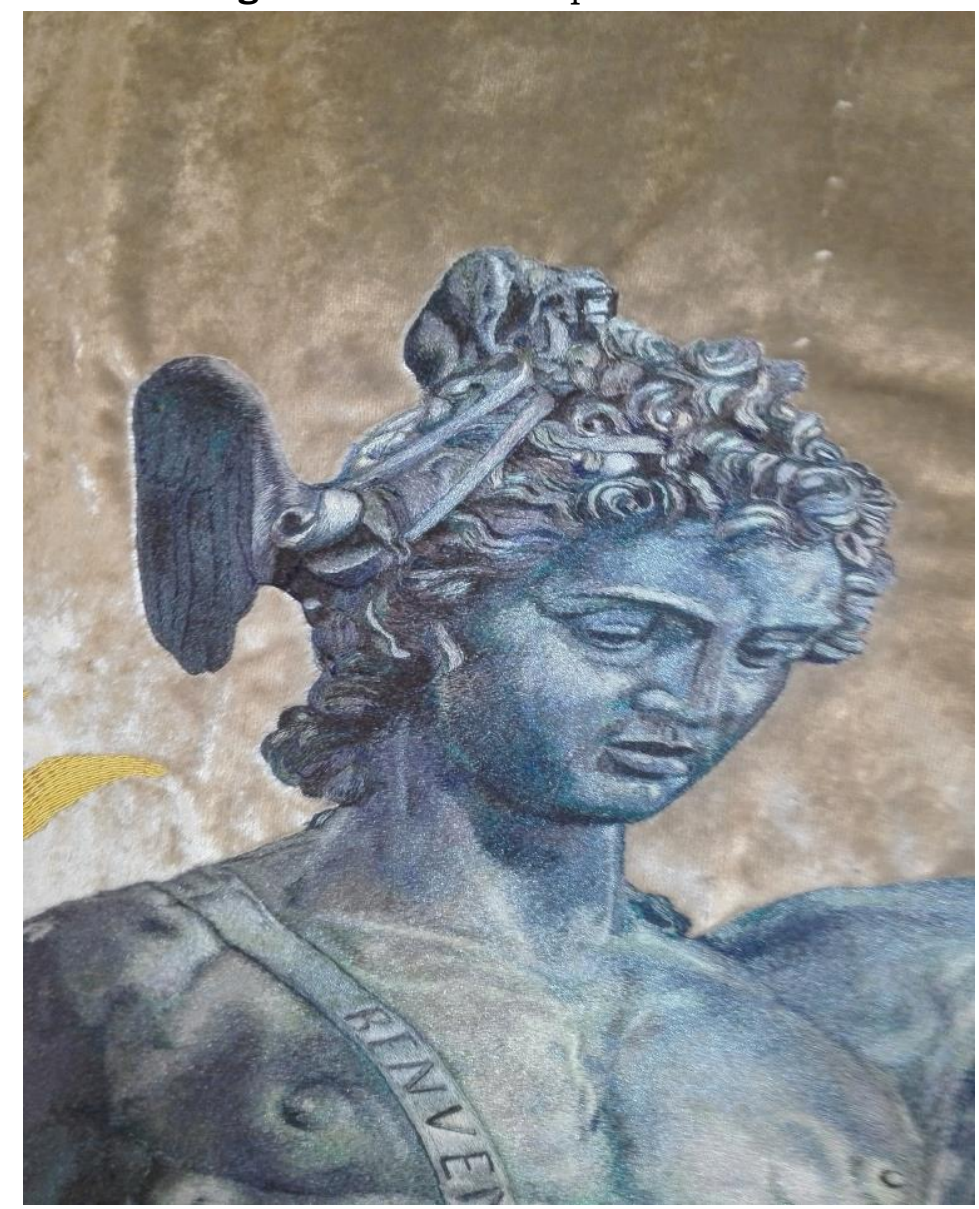

Fuente: Autores.

$\mathrm{El}$ arte del bordado encaja perfectamente en la definición de patrimonio cultural inmaterial, recogida en el artículo 2.1 de la Convención para la Salvaguardia del Patrimonio Cultural Inmaterial, pues este se define como "los usos, representaciones, expresiones, conocimientos y técnicas -junto con los instrumentos, objetos, artefactos y espacios culturales que les son inherentes- que las comunidades, los grupos y en algunos casos los individuos reconozcan como parte integrante de su patrimonio cultural. Este patrimonio cultural inmaterial, que se transmite de generación en generación, es recreado constantemente por las comunidades y grupos en función de su entorno, su interacción con la naturaleza y su historia, infundiéndoles un sentimiento de identidad y continuidad y contribuyendo así a promover el respeto de la diversidad cultural y la creatividad humana. A los efectos de la presente Convención, se tendrá en cuenta únicamente el patrimonio cultural inmaterial que sea compatible con los 
instrumentos internacionales de derechos humanos existentes y con los imperativos de respeto mutuo entre comunidades, grupos e individuos y de desarrollo sostenible".

La candidatura tiene el apoyo unánime de los lorquinos, de los pasos que conforman la Semana Santa y de la Comunidad Autónoma de la Región de Murcia. De ser incluida por la UNESCO en la Lista Representativa del Patrimonio Inmaterial de la Humanidad, dotaría a la ciudad de una nueva distinción competitiva que, teóricamente, repercutiría en el desarrollo turístico de manera positiva.

Finalmente, la última campaña publicitaria lanzada por el Ayuntamiento de Lorca hace una clara referencia a la Semana Santa y a sus bordados. El slogan Lorca, lo bordamos, creado en 2016, tiene como objetivo fundamental consolidarse como una marca de ciudad que no solo otorgue visibilidad a toda la actividad del municipio de cara al visitante, sino de la que también se sienta orgulloso el propio ciudadano, además de poner en valor los bordados e incluso otros símbolos de identidad representativos de Lorca como las distintas religiones que se asentaron en la ciudad (Cristianismo, Islam y Judía), la Torre Alfonsina del Castillo, la alfarería, la gastronomía, la ganadería, el sol y la playa (Imagen 3).

Imagen 3. Slogan Lorca, Lo bordamos

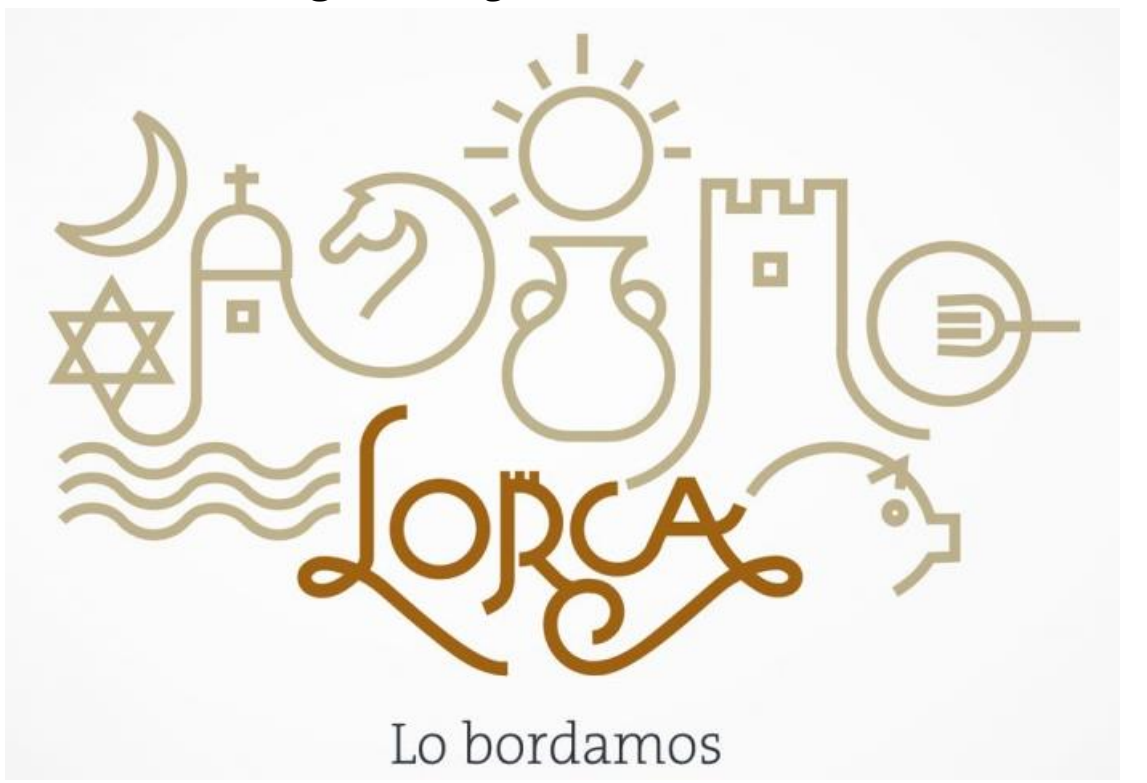

Fuente: Ayuntamiento de Lorca.

\section{TURISMO CULTURAL EN LORCA. ACCIONES EMPRENDIDAS Y SITUACIÓN ACTUAL}

La ciudad de Lorca se ha consolidado en los últimos años como el lugar regional donde más se ha apostado por el turismo cultural debido a su variado patrimonio, material e inmaterial. Los seísmos de 2011 marcan un antes y un después en el turismo y en el patrimonio lorquino (García, 2016). El desarrollo de esta modalidad tiene su inicio con el Plan de Desarrollo del Producto Cultural de la ciudad de Lorca (1997), promovido por la Dirección General de Economía y Planificación de la Consejería de Economía y Hacienda de la Región de Murcia, que inició las labores de recuperación y puesta en valor de su patrimonio para uso turístico (Troitiño, 2015).

No obstante, el impulso definitivo se produjo con la creación en 2003 del proyecto Lorca Taller del Tiempo, en el marco del Plan de Desarrollo Turístico de la Región de Murcia. El proyecto, en el que participan la administración local y regional y las organizaciones empresariales del municipio, fue diseñado para revalorizar el conjunto patrimonial lorquino, con un enfoque de apreciación turística y cultural desde la autenticidad, la calidad y la sostenibilidad. Se centra 
en la explotación de los valores y significado de los amplios recursos patrimoniales y culturales de la ciudad. Es decir, de acuerdo con García-Marín y Moreno-Muñoz (2017), pretende una serie de objetivos fundamentales:

- Revalorización del patrimonio cultural de Lorca y refuerzo de la identidad de sus habitantes.

- Potenciar el patrimonio cultural de Lorca, mediante mecanismos de protección, rehabilitación y mantenimiento del mismo.

- Dar valor a su riqueza histórica, artística y cultural, desarrollando una nueva imagen que sirve como polo de atracción turística.

- Fomentar el carácter didáctico del patrimonio lorquino como recurso educativo desde un punto de vista histórico y cultural.

- Generar una rentabilidad social, económica y cultural.

Desde su puesta en marcha, Lorca Taller del Tiempo supuso un impulso para el turismo en la ciudad. Una de las acciones con más aceptación entre los lorquinos y visitantes fue la restauración y adecuación del entorno de la Fortaleza del Sol. Desde su apertura al público en 2003 el número de visitantes ha ido variando (Gráfico 1). En términos absolutos se acumulan un total de 1.037 .504 visitantes, lo que arroja una media anual de 74.107. La última anualidad muestra un total de 79.924 visitas, número que se acerca al máximo alcanzado en 2010, justo antes de los seísmos. Los resultados más flojos (49.131 visitantes) coinciden con el año en el que sucedieron los terremotos, que afectaron a estructuras y obligó al cierre parcial. $\mathrm{El}$ remonte posterior también se relaciona con las rehabilitaciones (murallas y torre Alfonsina) y la apertura del parador (Cebrián, 2016). No obstante, son la mitad de los previstos inicialmente, lo que indica que el proyecto no está a pleno rendimiento pese a llevar tres lustros en activo.

Gráfico 1. Número de visitantes a la Fortaleza del Sol (2003-2016)

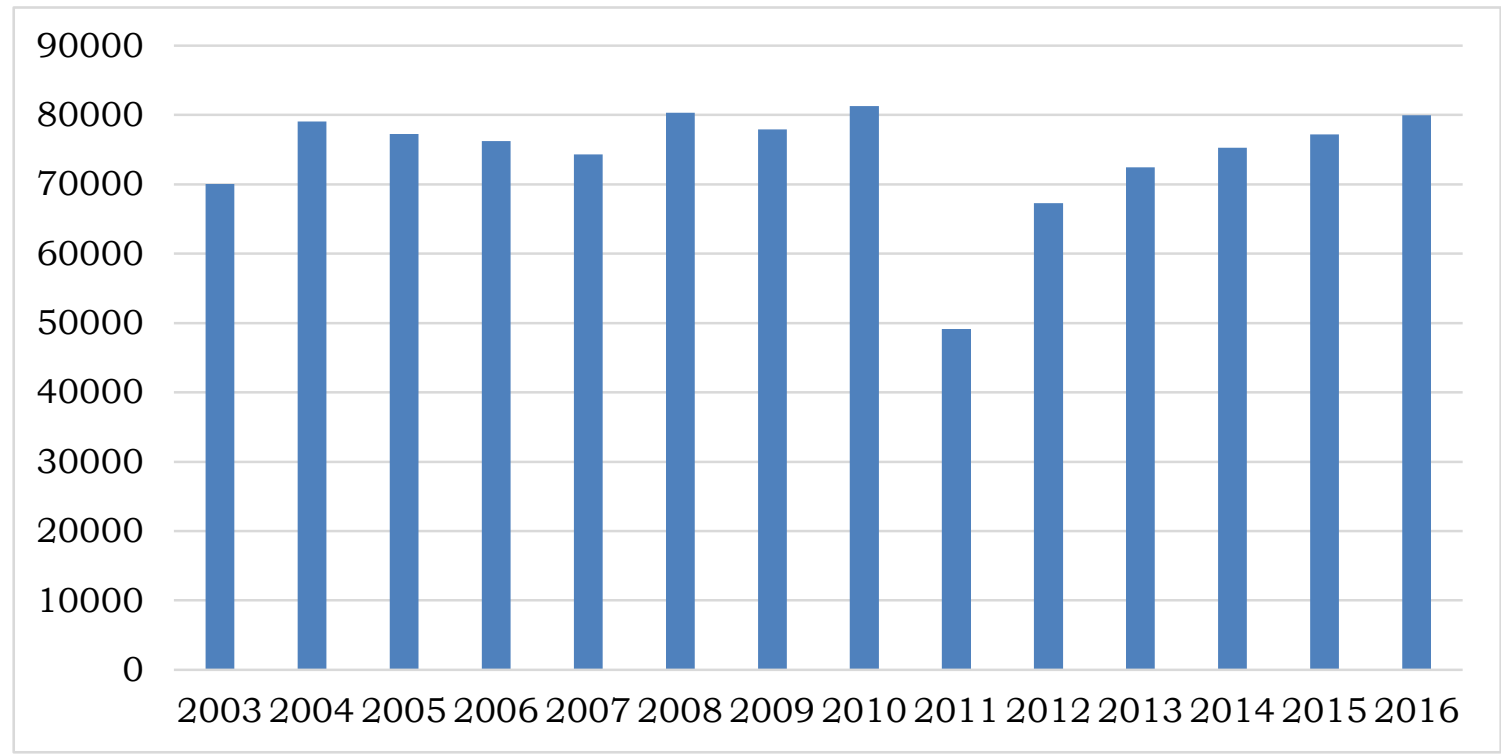

Fuente: Elaboración propia a través de los datos de Lorca Taller del Tiempo.

El 11 de mayo de 2011 más de medio centenar de inmuebles históricos se vieron dañados por los terremotos. Para atender a la urgencia de la restauración la Dirección General de Bellas Artes y Bienes Culturales del Ministerio de Cultura promovió el Plan Director de Recuperación del Patrimonio Cultural de Lorca (2011), con un plazo de ejecución de cinco años. Éste estuvo centrado fundamentalmente en la recuperación de los BIC, las viviendas emblemáticas del siglo 
XIX y comienzos del XX, así como en todo el caso histórico de la localidad. Por otro lado, dentro del propio Plan se creó una ruta denominada Lorca abierta por restauración, que mostró la adaptabilidad de la gestión (Cebrián, 2016) y que fue una mezcla de turismo cultural y el denominado dark tourism. La iniciativa consiguió un galardón durante la Feria Internacional del Turismo (FITUR) de 2012, concretamente el Premio Excelencias. Ese mismo año fue premiada con un "Global Awards" que la World Travel Market (WTM) concede anualmente a las mejores iniciativas del mundo en el sector turístico y con el que se reconocen los esfuerzos para la promoción y recuperación del patrimonio y la actividad turística local.

Conviene, en términos generales, analizar el número de viajeros en Lorca con el fin de conocer su tendencia. Tal como se observa en la Gráfico 2, durante el periodo $2000-2016$ se han sucedido varias etapas claramente diferenciadas. La primera comprende al lustro 2000-2004, periodo se pasa de los 61.150 viajeros en el año 2000 a los 70.235 de 2004, coincidiendo con la primera anualidad completa que abre sus puertas Lorca Taller del Tiempo. La segunda fase (2005-2009), muestra una fuerte regresión. Pese a que en 2005 apenas se nota el descenso de viajeros (69.388), en los siguientes años sí que se produce un gran descenso, especialmente entre 2007 y 2009, cuando se produce el mínimo de la serie (44.723) y la crisis económica global es más pronunciada. En 2010 se inicia una recuperación que se ve frenada con los terremotos de 2011, pues el registro de viajeros de 2012 es inferior al de 2011. Durante el último ciclo (2013-2016), el crecimiento anual es constante. Aumentándose el número de viajeros entre 2012 y 2016 en un 52,77\%. La última anualidad resalta, en valores absolutos, como el máximo histórico alcanzado en la serie con 71.708 viajeros.

Gráfico 2. Número de viajeros en Lorca (2000-2016)

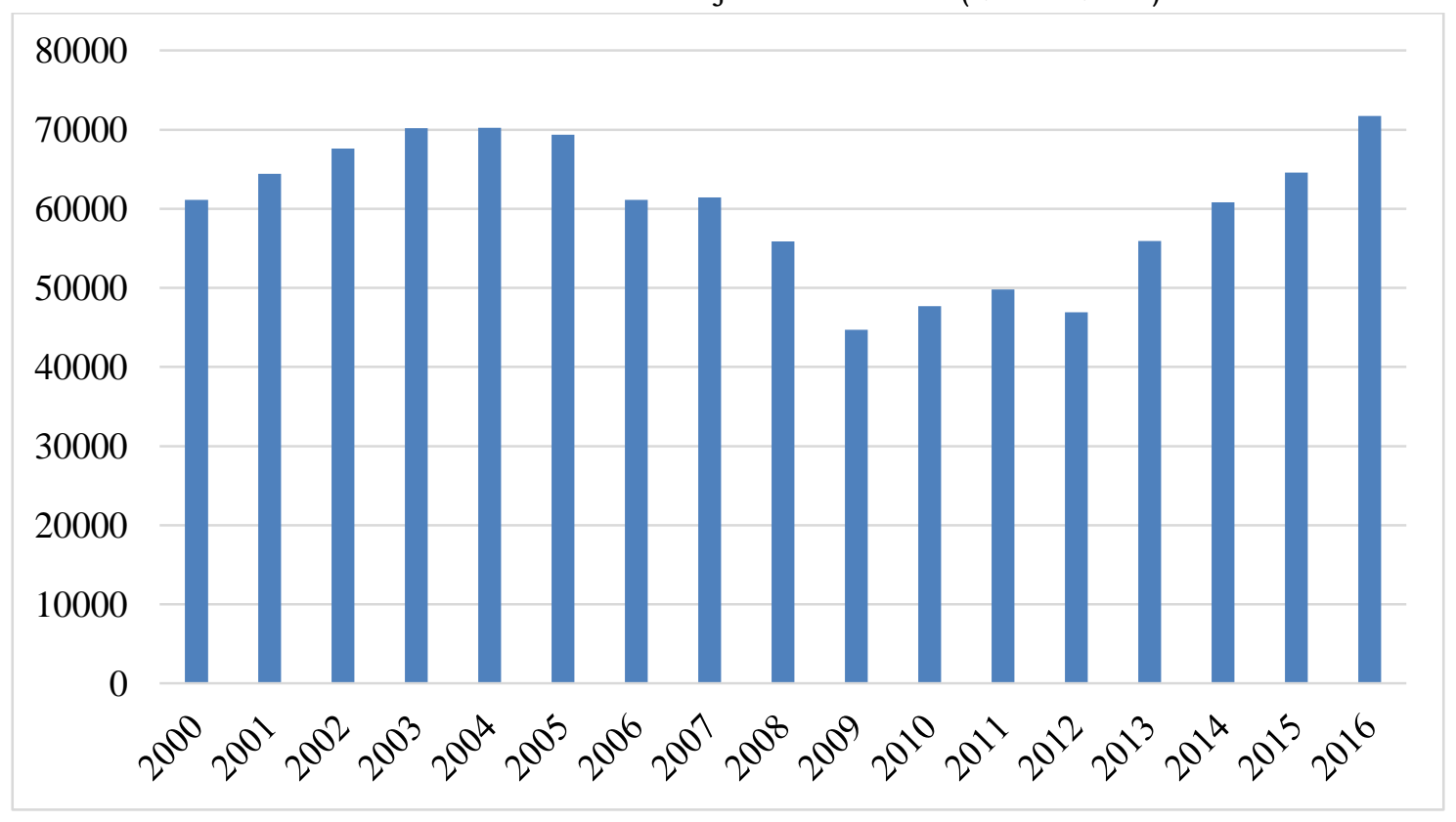

Fuente: Elaboración propia a través de los datos de MurciaTurística.

No obstante, si se comparan los datos de 2016 de Lorca con los de Murcia y Cartagena, ciudades regionales con amplia oferta de turismo cultural, se observan contrastes considerables. Murcia, por su condición de capital regional fue con diferencia la que mayor número de viajeros atrajo (430.315), mientras que Cartagena registró un total de 134.955, motivado en gran medida por el turismo de cruceros. Es decir, la ciudad de Lorca solamente aporta el 11,25 \% de los viajeros en las principales ciudades de turismo cultural en la Región de Murcia. 
En la actualidad se pueden realizar 5 rutas distintas por la ciudad, de corta duración y escasa dificultad, que pretenden acercar al visitante al patrimonio material e inmaterial y a la identidad de la ciudad. Son los siguientes:

- Lorca Monumental: Itinerario circular que se inicia en el Centro de Visitantes, donde se facilita la información sobre la diversidad patrimonial de la ciudad y sus servicios culturales y turísticos. Transcurre por el centro histórico, lugar en el que se localizan edificios del Renacimiento y el Barroco, destacando el Palacio de Guevara o "casa de las columnas”, máxima expresión del Barroco Lorquino (Segado, 2012), la Ex Colegiata San Patricio, construida en el siglo XVI y que es principal templo de la ciudad y el Conjunto Monumental de Santo Domingo, donde se encuentra el Museo de los Bordados del Paso Blanco.

- Via Crucis hasta el Mirador del Castillo: Se inicia en la Calle Nogalte, concretamente en la Iglesia de San Francisco. Este itinerario acerca al visitante a los museos de los bordados del Paso Azul y Paso Morado. Desde los Jardines y Ermitas de El Calvario se observa de manera panorámica el conjunto histórico de la ciudad, los Barrios Altos y el Castillo de Lorca.

- Barrio de San Cristóbal por el Puente de la Alberca: La ruta comienza en el antiguo Claustro del Convento de La Merced, desde donde se cruza el Rio Guadalentín por el Puente de La Alberca (1879). Este puente es una de las vías históricas de entrada a la ciudad, y la conecta con uno de sus barrios más tradicionales. Una vez llegado al Barrio de San Cristóbal se visita el Museo de Bordados del Paso Encarnado y la Iglesia de San Cristóbal.

- Castillo de Lorca por Santa Maria: El itinerario se inicia en pleno corazón de la ciudad, en la Plaza de España. En el ascenso hacia el Castillo se visita la Iglesia de Santa María, mezquita mayor de la ciudad islámica, desde la que se otea una de las mejores panorámicas de los Barrios Altos. La ruta finaliza en la Fortaleza del Sol, simbolo identitario Lorca.

- Santuario Virgen de las Huertas por Las Alamedas: Parte desde la Iglesia de San Mateo, en la céntrica calle Lope Gisbert. Desde allí el visitante se dirige hacia Las Alamedas, lugar donde se realiza un paseo por sus arbolados y jardines, cuya existencia se remonta al siglo XVIII. Tras ello, se llega al Partidor de los Tres Puentes, elemento esencial para el riego tradicional de la huerta lorquina. Por último, se visita el Santuario de Nuestra Señora Virgen de las Huertas, Patrona de Lorca.

A pesar de todo, sólo los nuevos hallazgos arqueológicos de la Judería y las rutas especiales diseñadas para conocer la trastienda de la Semana Santa y la recuperación del patrimonio de la ciudad parecen crear nuevos estímulos que atraigan a más visitantes, pero no es suficiente. Sin duda, el consorcio turístico supuso un revulsivo en la gestión del turismo en Lorca cuando se puso en marcha y en la recuperación de sus recursos patrimoniales, pero la ciudad necesita un empuje en lo que tiene que ver con su promoción turística a nivel nacional y la creación o redefinición de su producto turístico que le permita desarrollar nuevos nichos que la hagan salir de su estancamiento actual (Romera, 2016).

\section{IMPLICACIÓN DE LOS ACTORES LOCALES EN EL DESARROLLO TURÍSTICO}

Los actores locales juegan un papel decisivo a la hora de fomentar el desarrollo del turismo. Tal como indica Daher (2006), su grado de implicación es fundamental, ya que una buena predisposición por su parte confiere una mayor posibilidad de éxito al destino turístico. 
Al vertebrar Lorca sus planes turísticos en el turismo cultural es preciso conocer qué opinión tienen actores locales como comerciantes, hosteleros, administración pública y los ciudadanos, con el fin de constatar su implicación.

En primer lugar conviene plantear la cuestión de la implicación en el desarrollo turístico de la ciudad. El 84,6 \% de los entrevistados considera que están comprometidas en él. Lo achacan a diversos factores como la participación en actos culturales que se organizan, la involucración en las campañas publicitarias que organiza el Ayuntamiento, la Cámara de Comercio o Lorca Taller del Tiempo, difusión de los recursos patrimoniales a sus amistades de otras localidades regionales o de Comunidades Autónomas diferentes mediante el denominado "boca oído" y a través de las redes sociales que permiten compartir publicaciones relacionadas con la ciudad. Por otro lado, el 10,7 \% razona que no colabora en el crecimiento del turismo en Lorca, ya que se dedican tan solo a su actividad profesional, aunque sí que desean una mayor afluencia turistica por las repercusiones positivas que les acarrearia en los negocios. Mientras que el 4,7 $\%$ no se pronunció al respecto. Destaca la siguiente percepción:

"Por norma general me involucro bastante en el desarrollo del turismo en Lorca. Siempre intento participar en las actividades organizadas desde el consistorio e intento publicitar mis productos en internet. Mediante este último mecanismo también doy difusión a la ciudad, mostrando algunos monumentos como el Castillo"

Respecto a la incidencia del turismo cultural en la ciudad, casi un $70 \%$ de los encuestados (69,2\%), piensa que es un motor de desarrollo en Lorca. No obstante, tal como revela un comerciante, se debe seguir potenciando:

"El turismo cultural, especialmente desde la creación de Lorca Taller del Tiempo, ha supuesto una revitalización de la ciudad en materia turística. Desde mi punto de vista, las acciones de restauración del patrimonio han evitado pérdidas irreparables en monumentos que estaban en estado de decadencia. Gracias a las iniciativas culturales y de ocio creadas tras los terremotos se están produciendo numerosas visitas, que generan un mayor dinamismo en el centro histórico.

No obstante, considero que se puede fomentar aún más, pues las potencialidades son muy grandes"

Pese a ello, el 18,5 \% de los entrevistados creen que esta modalidad turística no ayuda al desarrollo de la ciudad, ni al aumento de las ventas en los distintos negocios del casco histórico. Esto queda constatado en la siguiente opinión:

"Es cierto que existe una mayor afluencia turística en Lorca y la reparación de monumentos ha sido evidente. Pero, el crecimiento de la ciudad hubiese sido el mismo. Muchos comerciantes seguimos teniendo las mismas ventas que hace 10 años, por lo que considero que no supone un mayor beneficio económico para los ciudadanos"

Por último, se mencionaron los saberes locales, especialmente los oficios artesanos, del municipio como un recurso turístico. Los encuestados han revelado que es necesario atraer el turismo hacia éstos, con el fin de ponerlos en valor. Entre las estrategias citadas para ello destacan las campañas publicitarias, señaladas por el 95,4 \%. Las consideran fundamentales, ya que los resultados obtenidos por las lanzadas por el Ayuntamiento y la Comunidad Autónoma han sido bastante satisfactorios. Por otro lado, también se han señalado la realización de actividades culturales (64,6 \%), la inserción en las políticas turísticas (57,7 \%), y las visitas guiadas a las instalaciones de oficios artesanales $(47,7 \%)$.

\section{CONCLUSIONES}

Lorca cuenta con una identidad patrimonial, especialmente ligada a sus bienes patrimoniales más emblemáticos. Elementos como el Castillo, la Ex - Colegiata San Patricio o la Semana Santa de Lorca, están interiorizados en ciudadanos como señas de identidad. Por ello, en los 
últimos años. en pos de consolidar su oferta turística, se han utilizado como principales reclamos para los turistas.

La implicación de los diferentes actores locales es inequivoca. Son plenamente conscientes de que juegan un papel decisivo a la hora de fomentar el turismo, pues éste debe ser promovido no solo por la Administración Pública, sino también por los propios ciudadanos, estén relacionados o no con la actividad turística. Comerciantes y hosteleros, principales beneficiarios del turismo en la ciudad, son los más involucrados en el cometido. Esto es debido, fundamentalmente, a que sus ventas se ven incrementadas con la afluencia turística de manera considerable. No obstante, la mejor época económica para ellos es la Semana Santa, dada la atracción generalizada del evento.

Lorca, al igual que otras ciudades históricas, tiene una ventaja cualitativa definida por la interdependencia que existe entre el patrimonio tangible e intangible. Ésta se ha consolidado en un instrumento esencial para la puesta en marcha del turismo cultural. Desde los terremotos de 2011 la incidencia de esta modalidad crece año tras año debido, fundamentalmente, a dos motivos: i) las enormes cantidades invertidas sobre el patrimonio dañado han conferido un nuevo atractivo a los lugares emblemáticos y, por ende, a la ciudad; y ii) Las campañas de publicidad han sido fructíferas, tal y como se desprenden del crecimiento del número de viajeros. Pero, el destino debe seguir potenciándose para poder competir con otras ciudades, pues la competencia en esta modalidad turística es constante. Por lo tanto, es ineludible continuar en la línea de trabajo actual, mediante fórmulas originales que se distingan de otros destinos turísticos culturales cercanos.

Por último, a modo de reflexión final, consideramos que el turismo continuará desarrollándose y utilizando como un instrumento de apoyo para la conservación del patrimonio y como herramienta para la promoción de identidad cultural.

\section{BIBLIOGRAFÍA}

Abdelmalki, I. y Courlet, C. (1996). Les nouvelles logiques du developpement. Paris, Francia: L'Harmattan.

Bowitz, E. y Ibenholt, K. (2009). Economic impacts of cultural heritage - Research and perspectives, Journal of Cultural Heritage, 10 (1), 1-8.

Bruzón, L. (2017). Audiovisual etnográfico y tradición: una contribución a la identidad y desarrollo del municipio, Universitas: Revista de Ciencias Humanas y Sociales, 27, 45-65.

Burghausen, M. y Balmer, J. (2014). Corporate heritage identity management and the multimodal implementation of a corporate heritage identity, Journal of Business Research, 67 (11), 2311-2323.

Capel, H. (2014). El patrimonio: la construcción del pasado y del futuro. Barcelona, España: Ediciones del Serbal.

Cebrián, A. (2016): Cebrián, A. (2016). El producto turístico de Lorca (Murcia, España): Políticas, resultados y perspectivas para destino turístico. En R. García (Ed.), Lorca: Ciudad Histórica del Mediterráneo (pp. 315-352). Murcia, España: Fundación Séneca Agencia de Ciencia y Tecnología de la Región de Murcia.

Daher, R.F. (2006): Urban Regeneration/Heritage Tourism Endeavours: The Case of Salt, Jordan "Local Actors, International Donors, and the State", International Journal of Heritage Studies, 11 (4), 289-308.

Espejo, C. y García, R. (2016). Los bordados de Lorca: Un patrimonio singular como recurso turístico. En R. García (Ed.), Lorca: Ciudad Histórica del Mediterráneo (pp. 175-206). Murcia, España: Fundación Séneca - Agencia de Ciencia y Tecnología de la Región de Murcia. 
García, R., Andrés, J.L. y Espejo, C. (2016): Conocimientos tácitos, transversalidad turística y desarrollo local. El ejemplo de una ciudad desolada y en proceso de reconversión: Lorca (Región de Murcia, España), Pasos: Revista de Turismo y Patrimonio Cultural, 14 (5), 1093-1107.

García-Marín, R. y Moreno-Muñoz, D. (2017). Patrimonio y turismo en la ciudad de Lorca: ¿Simbiosis sinérgica?, International Journal of Scientific Management and Tourism, 3 (1). 323-339.

Gil, A. (1968). La ciudad de Lorca (notas de Geografia Urbana), Papeles del Departamento de Geografía, 1, 79-110.

Henderson, J. (2010). Heritage, Identity and Tourism in Hong Kong, International Journal of Heritage Studies, 7 (3), 219-235.

Molano, O. L (2007). Identidad cultural: un concepto que evoluciona, Ópera, 7, 69-84.

Moreno-Muñoz, D., Garcia-Marin, R. y Espejo-Marin, C. (2017). A Semana Santa como fator de desenvolvimento turístico na cidade de Lorca (Região de Múrcia, Espanha). En F. Cravidão, N. Santos, C.Moreira, R. Ferreira, P. Nossa y L. Silveira, (Coords). Local identity and tourism management on world heritages sites. Trends and challenges (pp. 113-122). Coimbra, Portugal: Departamento de Geografia e Turismo, Universidade de Coimbra.

Prats, Ll. (2003). Patrimonio + turismo = ¿desarrollo?, Pasos: Revista de Turismo y Patrimonio Cultural, 1 (2), 127-136.

Richards, G. (1996). Production and consumption of european cultural tourism. Annals of Tourism Research, 23 (2), 261-283.

Segado, P. (2012). Lorca barroca. Murcia, España: Universidad de Murcia.

Serrano, J.M., Espejo, C., Andrés, J.L., y Romera, J.D. (2016). Patrimonio, turismo y desarrollo local en Lorca, Región de Murcia (España). En R. García, F. Alonso, F. Belmonte, y D. Moreno (Eds.). Actas del XV Coloquio Ibérico de Geografia: Retos y Tendencias de la Geografía Ibérica (822-831). Murcia, España: Asociación de Geógrafos Españoles.

Troitiño, L. (2015). La dimensión turística del patrimonio cultural de la ciudad de Lorca (Murcia, España), Cuadernos de Turismo, 36, 389-414.

Vecco, M. (2010). A definition of cultural heritage: From the tangible to the intangible, Journal of Cultural Heritage, 11, 321-324.

Zukin, S. (2012). The social production of urban heritage: Identity and ecosystem on an Amsterdam shopping street, City, Culture and Society, 3 (4), 281-291.

\section{AGRADECIMIENTOS}

La investigación ha sido financiada: Fundación Séneca, Agencia de Ciencia y Tecnología de la Región de Murcia bajo el Proyecto de Investigación: "El Turismo Cultural en la Revitalización del Patrimonio Histórico de Lorca: Actores y Estrategias" (Proyecto 18937/JLI/13). Agradecemos el apoyo prestado.

\section{HOW TO CITE THIS ARTICLE IN BIBLIOGRAPHIE}

Moreno Muñoz, D.M., García Marin, R., y Espejo Marin C. (2018): Identidad patrimonial e implicación local en el desarrollo turístico de Lorca (Región de Murcia, España) Rotur. Revista de Ocio y Turismo, 12(2): 16-29, http://www.rotur.es, ISSN: 1888-6884 